\title{
1 Direct Observation of Electrically Conductive Pili Emanating from
}

\section{Geobacter sulfurreducens}

3 Xinying Liu ${ }^{\mathrm{a}, \mathrm{b}}$, David J. F. Walker ${ }^{\mathrm{c}}$, Stephen S. Nonnenmann ${ }^{\mathrm{d}, \mathrm{e}}$, Dezhi Sun ${ }^{\mathrm{b}}$, Derek R. Lovley ${ }^{\mathrm{a}, \mathrm{d}}$

$4 \quad{ }^{\text {a }}$ Department of Microbiology, University of Massachusetts-Amherst, Amherst, Massachusetts, USA

$5 \quad{ }^{\mathrm{b}}$ College of Environmental Science and Engineering, Beijing Forestry University, Beijing, 100083, China

$6 \quad{ }^{\mathrm{c}}$ Institute for Cellular and Molecular Biology, University of Texas at Austin, Austin, Texas 78712, USA

$7 \quad{ }^{\mathrm{d}}$ Institute for Applied Life Sciences, university of Massachusetts_Amherst, Amherst, Massachusetts, USA

$8{ }^{\mathrm{e}}$ Department of Mechanical and Industrial Engineering, University of Massachusetts-Amherst, Amherst,

9 Massachusetts, USA

10 


\section{Abstract}

12 Geobacter sulfurreducens is a model microbe for elucidating the mechanisms for extracellular

13 electron transfer in several biogeochemical cycles, bioelectrochemical applications, and

14 microbial metal corrosion. Multiple lines of evidence previously suggested that electrically

15 conductive pili (e-pili) are an essential conduit for long-range extracellular electron transport in

16 G. sulfurreducens. However, it has recently been reported that G. sulfurreducens does not

17 express e-pili and that filaments comprised of multi-heme $c$-type cytochromes are responsible for

18 long-range electron transport. This possibility was directly investigated by examining cells,

19 rather than filament preparations, with atomic force microscopy. Approximately $90 \%$ of the

20 filaments emanating from wild-type cells had a diameter $(3 \mathrm{~nm})$ and conductance consistent with

21 previous reports of e-pili harvested from $G$. sulfurreducens or heterologously expressed in E. coli

22 from the G. sulfurreducens pilin gene. The remaining $10 \%$ of filaments had a morphology

23 consistent with filaments comprised of the $c$-type cytochrome OmcS. A strain expressing a

24 modified pilin gene designed to yield poorly conductive pili expressed $90 \%$ filaments with a 3

$25 \mathrm{~nm}$ diameter, but greatly reduced conductance, further indicating that the $3 \mathrm{~nm}$ diameter

26 conductive filaments in the wild-type strain were e-pili. A strain in which genes for five of the

27 most abundant outer-surface $c$-type cytochromes, including OmcS, was deleted yielded only 3

$28 \mathrm{~nm}$ diameter filaments with the same conductance as in the wild-type. These results demonstrate

29 that e-pili are the most abundant conductive filaments expressed by G. sulfurreducens, consistent

30 with previous functional studies demonstrating the need for e-pili for long-range extracellular

31 electron transfer. 


\section{Importance}

35 Electroactive microbes have significant environmental impacts as well as applications in

36 bioenergy and bioremediation. The composition, function, and even existence of electrically

37 conductive pili (e-pili) has been one of the most contentious areas of investigation in

38 electromicrobiology, in part because e-pili offer a mechanism for long-range electron transport

39 that does not involve the metal co-factors common in much of biological electron transport. This

40 study demonstrates that e-pili are abundant filaments emanating from Geobacter sulfurreducens,

41 which serves as a model for long-range extracellular electron transfer in direct interspecies

42 electron transfer, dissimilatory metal reduction, microbe-electrode exchange, and corrosion

43 caused by direct electron uptake from $\mathrm{Fe}(0)$. The methods described in this study provide a

44 simple strategy for evaluating the distribution of conductive filaments throughout the microbial

45 world with an approach that avoids artifactual production and/or enrichment of filaments that

46 may not be physiologically relevant. 
Electroactive microorganisms are important in multiple biogeochemical cycles, the human

50 gut, several bioenergy strategies, and metal corrosion $(1,2)$. One of the most contentious issues

51 in electromicrobiology has been the role of electrically conductive protein nanowires in

52 facilitating long-range electron transport. Electrically conductive protein nanowires have been

53 studied most extensively in Geobacter sulfurreducens, which has served as the model microbe

54 for elucidating the mechanisms of long-range electron transport in Geobacter species (3).

55 Geobacter are of interest because they are often the most abundant electroactive microbes in

56 soils and sediments in which organic matter oxidation is coupled to Fe(III) oxide reduction; in

57 natural methanogenic environments and anaerobic digesters where they serve as electron-

58 donating partners for direct interspecies electron transfer (DIET) with methanogens; and in

59 electrode biofilms harvesting electricity from waste organic matter (3-5). Furthermore,

60 Geobacter are the most effective microbes available in culture for extracellular electron transport

61 functions such as Fe(III) oxide reduction (3), producing electric current (5), DIET (6), and

62 corrosion via direct extraction of electrons from metallic iron $(7,8)$. An additional area of

63 interest is the potential for constructing electronic devices with novel functions with $G$.

64 sulfurreducens protein nanowires (9).

65 Debate has arisen over the composition of G. sulfurreducens protein nanowires and their

66 role in long-range electron transfer. Multiple lines of evidence have suggested that electrically

67 conductive pili (e-pili) are the most abundant G. sulfurreducens protein nanowires and that e-pili

68 are essential for long-range electron transport $(10,11)$. However, two recent publications have

69 suggested that G. sulfurreducens does not express e-pili and that protein nanowires comprised of

70 the multi-heme $c$-type cytochromes OmcS and OmcZ are the functional conduits for long-range

71 extracellular electron transfer $(12,13)$. The primary argument against the production of e-pili is 
72 the fact filaments comprised of $c$-type cytochromes are the most abundant filaments observed in

73 filament preparations observed with cryo-electron microscopy $(12,13)$. However, generating

74 these filament preparations involves shearing filaments from the cell, purifying the filaments

75 under high $\mathrm{pH}$, selective precipitation with ammonium sulfate, and affixing filaments to grids.

76 Each of these steps has the potential to selectively enrich specific filaments or for artifactual

77 formation of cytochrome filaments (11). For example, in studies of G. sulfurreducens filament

78 preparations prepared by the same person in the same laboratory, under identical conditions,

79 cryo-electron microscopy suggest that a majority of the filaments were comprised of OmcS (14),

80 whereas filaments with a diameter consistent with e-pili, not OmcS, were observed with atomic

81 force microscopy (AFM) (15).

\section{Direct AFM of Cells.}

83 In order to avoid potential artifacts/enrichments associated with filament purification, the

84 filaments associated with G. sulfurreducens cells were directly examined with AFM. To simplify

85 the analysis, cells were grown with fumarate as the electron acceptor, a growth condition in

86 which the pilin monomer PilA and OmcS are expressed, but expression of the gene for the multi-

87 heme cytochrome OmcZ is repressed (16-18). AFM of culture aliquots directly deposited on a

88 conductive surface revealed cells with abundant filaments (Fig. 1A and Supplemental Fig. S1A).

89 There were two types of filaments emanating from the cells. One filament type appeared to be

90 comprised of OmcS, as evidenced from its $4 \mathrm{~nm}$ diameter (Fig. 1B and Supplemental Fig. S1B)

91 and its characteristic axial periodicity with a $20 \mathrm{~nm}$ pitch $(12,14)$ (Fig. 1C). The OmcS

92 filaments consistently accounted for only ca. $10 \%$ of the filaments observed (Fig.1A and

93 Supplemental Figs. S2-4). 
94 Approximately $90 \%$ of the filaments were $3 \mathrm{~nm}$ in diameter (Fig. 1A and Supplemental

95 Figs. S2-4), the same diameter as the filaments observed when the G. sulfurreducens PilA gene

96 is expressed in Pseudomonas aeruginosa (19) or Escherichia coli (20) and the same diameter of

97 individual conductive filaments previously harvested from $G$. sulfurreducens $(16,21)$. These

98 results suggest that the $3 \mathrm{~nm}$ diameter filaments are e-pili. As expected from the growth

99 conditions employed, no filaments with a morphology consistent with the $2.5 \mathrm{~nm}$ diameter and

100 axial pitch of OmcZ filaments (13) were observed. Both the OmcS and e-pili filaments exhibited

101 an ohmic-like response (Fig. 1D). The conductance of the e-pili was slightly higher than that of

102 the OmcS filaments (Fig. 1D). 

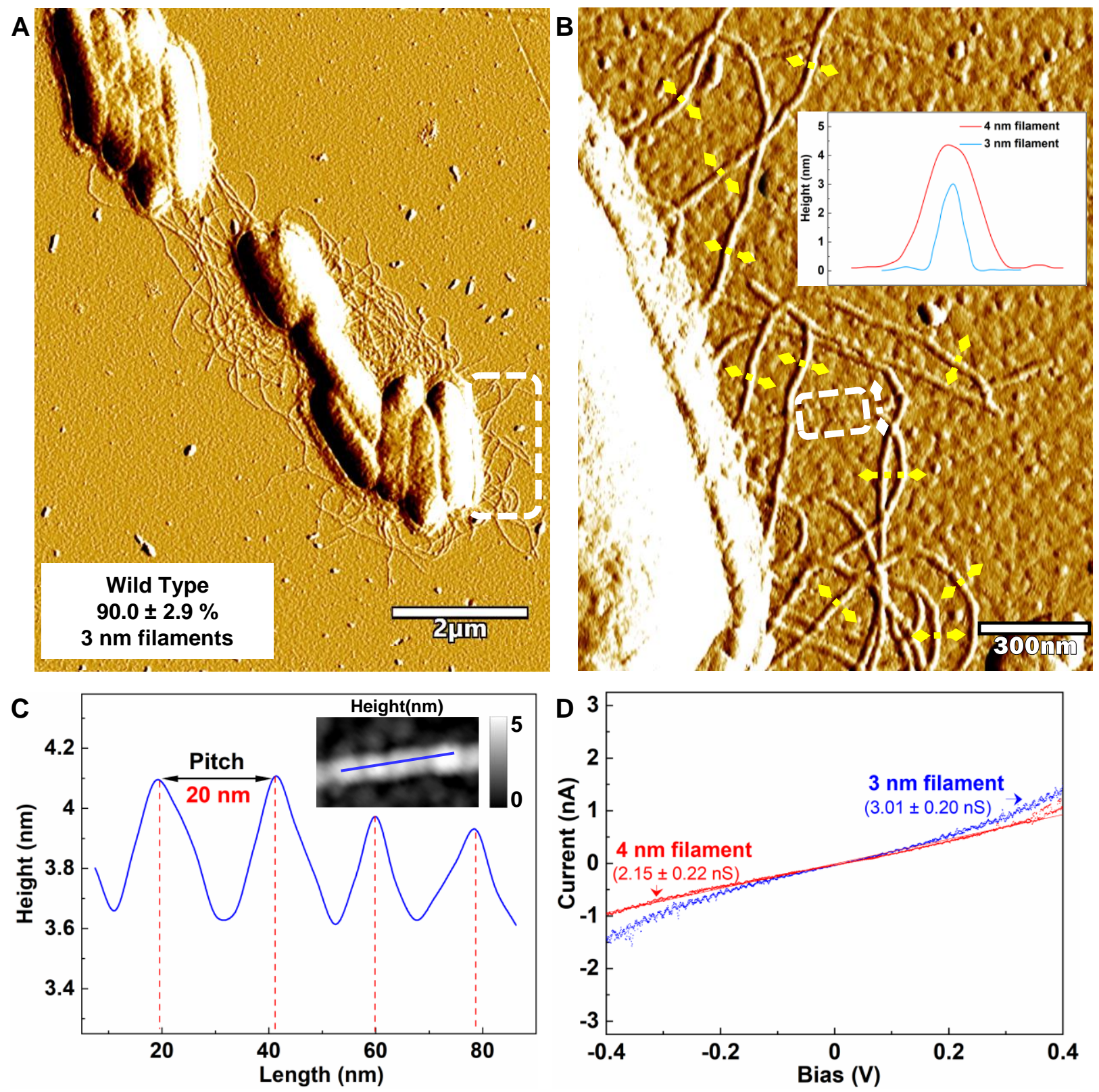

Fig. 1. Characterization of filaments emanating from $G$. sulfurreducens with the wild-type pilin gene. (A) AFM amplitude image. The proportion of $3 \mathrm{~nm}$ diameter filaments was calculated from the total number of $3 \mathrm{~nm}$ and $4 \mathrm{~nm}$ diameter filaments counted in 9 regions from 3 separate samples (Supplemental Figs. 2-4) and were determined from height images similar to those shown in Supplemental Fig. 1. (B) Higher magnification of the region highlighted in the dashed frame in panel A. Inset shows typical height profiles across the 3 $\mathrm{nm}$ (yellow lines) and $4 \mathrm{~nm}$ (white line) diameter filaments, as determined from the corresponding height images (Supplemental Fig. S1B). Due to fluctuation of diameter along the axis of the filaments, diameters were measured at the points of greatest diameter for consistency. (C) Longitudinal height profile (along solid blue line in inset) for region on the $4 \mathrm{~nm}$ filament noted by the white dashed frame in panel B. (D) Comparison of point-mode current response (I-V) spectroscopy for $4 \mathrm{~nm}$ (red) and $3 \mathrm{~nm}$ (blue) diameter filaments. 
116 The responses shown are representative of three different measurements on each of three 117 individual filaments (Supplemental Figs. S5 and S6). Conductance (mean + standard 118 deviation, $\mathbf{n = 9}$ ) was calculated from a linear fit model between $\mathbf{- 0 . 2} \mathrm{V}$ and $0.2 \mathrm{~V}$ 119 (Supplemental Figs. S5 and S6).

G. sulfurreducens strain Aro-5 was previously constructed to replace the PilA pilin gene

122 with aro-5, a synthetic pilin gene designed to yield poorly conductive pili (18). The conductivity

123 of filaments harvested from the cells is much lower than the conductivity of filaments harvested

124 from wild-type controls $(18,21-23)$. Direct examination of filaments emanating from strain Aro-

1255 revealed two types of filaments, morphologically similar to those observed in the wild-type

126 control (Figs. 2A, B and Supplemental Fig. S1C, D). Filaments with a diameter and longitudinal

127 pitch (Fig. 2C) consistent with OmcS filaments comprised ca. $10 \%$ of the filaments (Fig. 2A,

128 Supplemental Figs. S7 and S8), similar to the OmcS filament abundance in the wild-type control

129 and consistent with the observation that strain Aro-5 produces abundant OmcS (18). The

130 conductance of these $4 \mathrm{~nm}$ diameter filaments was the same as the conductance observed for the

131 OmcS filaments of the wild-type control (Fig. 2D and Supplemental Fig. S9). As with the wild-

132 type strain, the $3 \mathrm{~nm}$ diameter filaments accounted for ca. $90 \%$ of the filaments observed, but

133 their conductance was more than 100-fold lower (Fig. 2D and Supplemental Fig. S10). This

134 decreased conductance is in agreement with previous observations of attenuated conductivity in

135 filaments harvested from strain Aro-5, including measurements on individual $3 \mathrm{~nm}$ diameter

136 filaments $(18,21-23)$. The dramatic change in the conductance of the $3 \mathrm{~nm}$ filaments emanating

137 from cells associated with the expression of aro-5 pilin gene provides further evidence that the 3

138 nm filaments in the wild-type strain were e-pili.

139 In order to further investigate the possibility of cytochrome-based filaments, we next

140 examined the previously described strain $\triangle$ omcBESTZ (24) in which the genes for the most

141 abundant $G$. sulfurreducens outer surface multi-heme c-type cytochromes, OmcB, OmcE, OmcS, 
142 OmcT, and OmcZ were deleted. As expected, filaments with morphologies consistent with

143 OmcS-based filaments were not apparent in this strain. All of the filaments emanating from

144 strain $\triangle$ omcBESTZ and lying near the cells were short, but had a diameter of $3 \mathrm{~nm}$ (Fig. 2E, F

145 and Supplemental Fig. S1E, F). Their conductance was the same as for the $3 \mathrm{~nm}$ filaments of the

146 wild-type strain (Fig. 2H, Supplemental Fig. S11). 

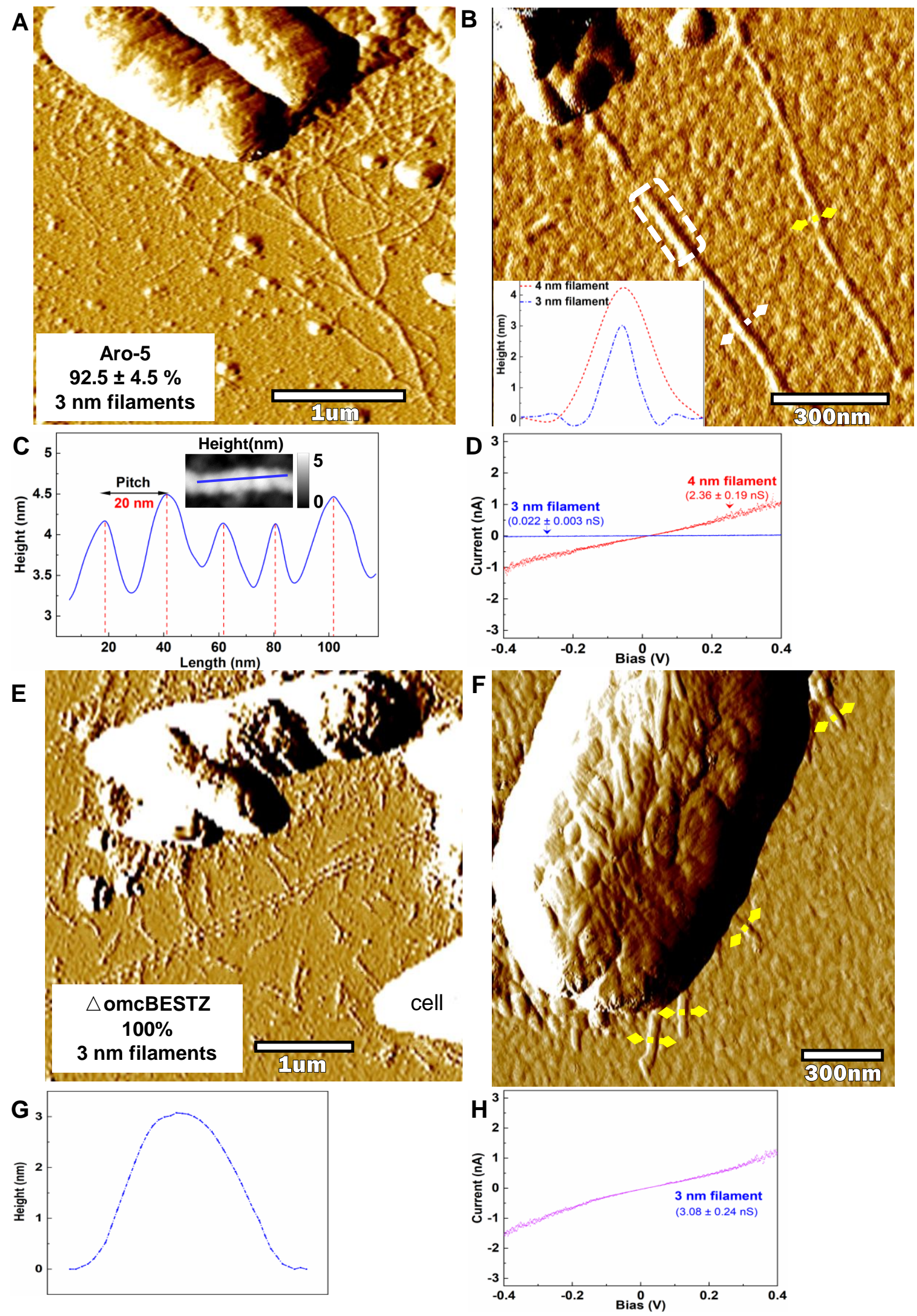
Fig. 2. Characterization of filaments emanating from G. sulfurreducens strain Aro-5 and strain $\triangle$ omcBESTZ. (A) AFM amplitude image of filaments associated with strain Aro-5. The proportion of $3 \mathrm{~nm}$ diameter filaments was calculated from the total number of $3 \mathbf{~ n m}$ and $4 \mathrm{~nm}$ diameter filaments counted in 6 regions from 3 separate samples (Supplemental Figs. S7 and S8) and were determined from height images similar to those shown in Supplemental Fig. 1. (B) AFM amplitude image at higher magnification illustrating the two filament types. Inset shows typical height profiles across the $3 \mathrm{~nm}$ (yellow lines) and $4 \mathrm{~nm}$ (white line) diameter filaments, as determined from the corresponding height images (Supplementary Fig. 1D). (C) Longitudinal height profile (along solid blue line in inset) for the portion of the $4 \mathbf{~ n m}$ diameter filament within the white frame in panel $B$. (D) Comparison of point-mode current response (I-V) spectroscopy for $4 \mathrm{~nm}$ (red) and $3 \mathrm{~nm}$ (blue) filaments. The responses shown are representative of three different measurements on three individual wires (Supplemental Figs. S9 and S10). Conductance (mean + standard deviation, $n=9$ ) was calculated from a linear fit model between $-0.2 \mathrm{~V}$ and $0.2 \mathrm{~V}$ (Supplemental Figs. S9 and S10). (E) AFM amplitude image of filaments associated with strain $\triangle$ omcBESTZ. (F) AFM amplitude image at higher magnification showing $3 \mathbf{~ n m}$ diameter filaments emanating from cell of strain $\triangle$ omcBESTZ. (G) Typical height profile across the filaments designated by yellow lines in panel $F$, as determined from the corresponding height images (Supplementary Fig. 1F). (H) Point-mode current response (IV) spectroscopy representative of three different measurements on three individual wires (Supplemental Fig. S11) on $3 \mathrm{~nm}$ filaments emanating from strain $\triangle$ omcBESTZ. Conductance (mean + standard deviation, $n=9$ ) was calculated from a linear fit model between $-0.2 \mathrm{~V}$ and $0.2 \mathrm{~V}$ (Supplemental Fig. S11).

Implications.

The results of direct observation of filaments emanating from cells of G. sulfurreducens demonstrates that $G$. sulfurreducens copiously expresses filaments with properties expected for e-pili. The e-pili were ca. 10-fold more abundant than putative OmcS filaments. These observations are in accordance with a number of previous observations. For example, when a pilin monomer modified with a peptide tag was expressed in G. sulfurreducens all of the filaments observed emanating from the cells were also decorated with the peptide tag (25). Several studies reported recovery of electrically conductive $3 \mathrm{~nm}$ diameter filaments when filaments were sheared off the outer surface of $G$. sulfurreducens $(16,21,25)$ or when the $G$. sulfurreducens pilin monomer was expressed in P. aeruginosa (19) or E. coli (20). Furthermore, as shown here, expressing aro-5 instead of PilA resulted in $3 \mathrm{~nm}$ filaments emanating from the cells with a similar morphology, but greatly attenuated conductance. Heterologously expressing 
184 a pilin gene encoding increased aromatic amino acid content yielded $3 \mathrm{~nm}$ diameter filaments

185 with 5000-fold higher conductivity than the wild-type control (26). These results are consistent

186 with the expression of e-pili and inconsistent with cytochrome-based filaments, as was the

187 finding reported here that the $3 \mathrm{~nm}$ filaments were still produced in a strain in which the genes

188 for all the most abundant outer-surface cytochromes were deleted. The abundance of e-pili in $G$.

189 sulfurreducens is also consistent with the finding that microbes that do not express outer-surface

$190 c$-type cytochromes can construct conductive filaments from monomers homologous to the $G$.

191 sulfurreducens pilin monomer $(22,23,27)$.

192 Notably, G. sulfurreducens strains that express pili of low conductance are consistently

193 deficient in long-range extracellular electron transfer $(18,22,28)$, providing strong evidence for

194 the role of e-pili in extracellular electron transport. The same cannot be said of the cytochrome

195 filaments OmcS and OmcZ. G. sulfurreducens strain Aro-5 cannot produce highly conductive

196 biofilms or high current densities on anodes (18), whereas deleting omcS has no impact on these

197 phenotypes $(17,29)$. Deletion of $o m c S$ can inhibit Fe(III) oxide reduction, in some, but not all

198 variants of $G$. sulfurreducens $(30,31)$. When deletion of omcS does have an impact, the strain

199 can be rescued for $\mathrm{Fe}(\mathrm{III})$ oxide reduction with the addition of ultrafine-grained magnetite (32).

200 However, magnetite cannot substitute for e-pili, demonstrating an essential role for e-pili in

201 Fe(III) oxide reduction, but not for OmcS. OmcZ is not required for Fe(III) oxide reduction (17)

202 and is not highly expressed in cells reducing Fe(III) oxide (30). Although it was suggested that

203 OmcZ filaments might account for the high conductivity of anode biofilms (13), this hypothesis

204 is inconsistent with the poor current production by strain Aro-5 and the low conductivity of its

205 biofilms (18). Furthermore, OmcZ is localized near the anode-biofilm interface, OmcZ filaments

206 are not observed coursing through the bulk of the biofilm (33). 
208 has demonstrated that G. sulfurreducens expresses e-pili in abundance, consistent with multiple

209 lines of evidence from previous studies $(10,11)$ that have indicated that $G$. sulfurreducens e-pili

210 are an important component in long-range extracellular electron transport. The cells examined

211 produced few OmcS-based filaments. The physiological significance of cytochrome-based

212 filaments is yet to be determined.

213 


\section{Methods}

\section{Culture source and growth conditions.}

216 The strain of G. sulfurreducens expressing wild-type PilA pilin gene as well as strain Aro-5,

217 which expresses a synthetic pilin gene designed to yield poorly conductive pili, and strain

$218 \Delta$ omcBESTZ, which features deletions in five major outer-surface $c$-type cytochromes, were

219 obtained from our laboratory culture collection and were previously described in detail $(18,24)$.

220 Cells were grown in medium with acetate $(10 \mathrm{mM})$ as electron donor and fumarate $(40 \mathrm{mM})$ as

221 electron acceptor as previously described (34). Cultures for AFM analysis were grown in the

222 acetate-fumarate medium at $25^{\circ} \mathrm{C}$, a condition known to promote expression of e-pili (16).

\section{Analysis with atomic force microscopy.}

224 An aliquot $(50 \mu \mathrm{l})$ of culture was drop cast onto a silicon wafer coated with a 35 nm layer of

225 platinum, prepared as previously described (35). After $12 \mathrm{~min}$, excess liquid was removed with a

226 pipette and the substrate was washed twice with $50 \mu 1$ of deionized water. Excess water was

227 absorbed with filter paper and the preparation was allowed to air dry. Samples were equilibrated

228 at $40 \%$ humidity inside scanning chamber of a Cypher ES, atomic force microscope (Asylum

229 Research, Oxford Instrument) for at least $1 \mathrm{~h}$ at $25^{\circ} \mathrm{C}$. The filaments were first observed with

230 tapping mode (AC-air topography) under repulsive force with a Pt/Ir-coated tip (PtSi-FM,

231 NanoWorld AG) at a $\sim 2.0 \mathrm{~N} / \mathrm{m}$ spring force constant and $\sim 70 \mathrm{kHz}$ resonance frequency.

232 The conductance of individual filaments was determined in contact mode (force $30 \mathrm{nN}$ )

233 with the Pt/Ir-coated tip functioning as the translatable top electrode. Quadruplicate amplitude of

$234 \pm 0.4 \mathrm{~V}$ voltage at $0.99 \mathrm{~Hz}$ frequency was applied to get ca.8000 points per measurement. Three

235 independent points from three individual wire (biological replicates) were analyzed to determine 
bioRxiv preprint doi: https://doi.org/10.1101/2021.07.06.451359; this version posted July 7, 2021. The copyright holder for this preprint (which was not certified by peer review) is the author/funder. All rights reserved. No reuse allowed without permission.

236 the conductance. Conductance was calculated from the linear slope between -0.2 to $0.2 \mathrm{~V}$

237 followed with the equation: Conductance $=$ Current/Voltage as preciously described (23). 


\section{References}

239 1. Shi L, Dong H, Reguera G, Beyenal H, Lu A, Liu J, Yu H-Q, Fredrickson JK. 2016.

$240 \quad$ Extracellular electron transfer mechanisms between microorganisms and minerals. Nature Reviews Microbiology 14:651-662.

2. Lovley DR, Holmes DE. 2021. Electromicrobiology: The ecophysiology of phylogenetically diverse electroactive microorganims. Nature Reviews Microbiology 19:(in press).

3. Lovley DR, Ueki T, Zhang T, Malvankar NS, Shrestha PM, Flanagan K, Aklujkar M, Butler JE, Giloteaux L, Rotaru A-E, Holmes DE, Franks AE, Orellana R, Risso C, Nevin KP. 2011. Geobacter: the microbe electric's physiology, ecology, and practical applications. Adv Microb Physiol 59:1-100.

4. Zhao Z, Li Y, Zhang Y, Lovley DR. 2020. Sparking anaerobic digestion: promoting direct interspecies electron transfer to enhance methane production. iScience 23:101794.

5. Logan BE, Rossi R, Ragab A, Saikaly PE. 2019. Electroactive microorganisms in bioelectrochemical systems. Nature Reviews Microbiology 17:307-319.

6. Lovley DR. 2017. Syntrophy goes electric: direct interspecies electron transfer. Ann Rev Microbiol 71:643-664.

7. Tang H-Y, Yang C, Ueki T, Pittman CC, Xu D, Woodard TL, Holmes DE, Gu T, Wang F, Lovley DR. 2021. Direct metal-microbe electron transfer is required for microbial corrosion of stainless steel. ISME J 15:https://doi.org/10.1038/s41396-021-00990-2.

8. Tang H-Y, Holmes DE, Ueki T, Palacios PA, Lovley DR. 2019. Iron corrosion via direct metal-microbe electron transfer. mBio 10:e00303-19.

9. Lovley DR, Yao J. 2021. Intrinsically conductive microbial nanowires for 'green' electronics with novel functions. Trends in Biotechnology

https://doi.org/10.1016/j.tibtech.2020.12.005

10. Lovley DR, Holmes DE. 2020. Protein Nanowires: The Electrification of the Microbial World and Maybe Our Own. J Bacteriol 202:e00331-20.

11. Lovley DR, Walker DJF. 2019. Geobacter protein nanowires. Frontiers in Microbiology 10:2078.

12. Wang F, Gu Y, O'Brien JP, Yi SM, Yalcin SE, Srikanth V, Shen C, Vu D, Ing NL, Hochbaum AI, Egelman EH, Malvankar NS. 2019. Structure of microbial nanowires reveals stacked hemes that transport electrons over micrometers. Cell 177:361-369.

13. Yalcin SE, O’Brien JP, Gu Y, Reiss K, Yi SM, Jain R, Srikanth V, Dahl DJ, Huynh W, Vu D, Acharya A, Chaudhuri S, Varga T, Batista VS, Malvankar NS. 2020. Electric field stimulates production of highly conductive microbial OmcZ nanowires. Nature Chemical Biology 16:1136-1142.

14. Filman DJ, Marino SF, Ward JE, Yang L, Mester Z, Bullitt E, Lovley DR, Strauss M. 2019. Cryo-EM reveals the structural basis of long-range electron transport in a cytochrome-based bacterial nanowire. Communications Biology 2:219.

15. Fu T, Liu X, Gao H, Ward JE, Liu X, Yin B, Wang Z, Zhuo Y, Walker DJF, Yang J, Chen J, Lovley DR, Yao J. 2020. Bioinspired bio-voltage memristors. Nature Communications 11:1861.

281

16. Reguera G, McCarthy KD, Mehta T, Nicoll JS, Tuominen MT, Lovley DR. 2005. Extracellular electron transfer via microbial nanowires. Nature 435:1098-1101. 
17. Nevin KP, Kim B-C, Glaven RH, Johnson JP, Woodard TL, Methé BA, DiDonato Jr RJ, Covalla SF, Franks AE, Liu A, Lovley DR. 2009. Anode biofilm transcriptomics reveals outer surface components essential for high current power production in Geobacter sulfurreducens fuel cells. PLoS ONE 4:e5628.

18. Vargas M, Malvankar NS, Tremblay P-L, Leang C, Smith JA, Patel P, Snoeyenbos-West O, Nevin KP, Lovley DR. 2013. Aromatic amino acids required for pili conductivity and long-range extracellular electron transport in Geobacter sulfurreducens mBio 4:e010513. .

19. Liu X, Wang S, Xu A, Zhang L, Liu H, Ma LZ. 2019. Biological synthesis of highconductive pili in aerobic bacterium Pseudomonas aeruginosa. Appl Microbiol Biotechnol 103:1535-1544.

20. Ueki T, Walker DJF, Woodard TL, Nevin KP, Nonnenmann S, Lovley DR. 2020. An Escherichia coli chassis for production of electrically conductive protein nanowires. ACS Synthetic Biology 9:647-654.

21. Adhikari RY, Malvankar NS, Tuominen MT, Lovley DR. 2016. Conductivity of individual Geobacter pili. RSC Advances 6:8354-8357.

22. Walker DJF, Adhikari RY, Holmes DE, Ward JE, Woodard TL, Nevin KP, Lovley DR. 2018. Electrically conductive pili from genes of phylogenetically diverse microorganisms. ISME J 12:48-58.

23. Walker DJF, Martz E, Holmes DE, Zhou Z, Nonnenmann SS, Lovley DR. 2019. The archaellum of Methanospirillum hungatei is electrically conductive. mBio 10:e00579-19.

24. Voordeckers JW, Izallalen M, Kim B-C, Lovley DR. 2010. Role of Geobacter sulfurreducens outer surface $c$-type cytochromes in the reduction of soil humic acid and the humics analog anthraquinone-2,6-disulfonate. Appl Environ Microbiol 76:2371-2375.

25. Ueki T, Walker DJF, Tremblay P-L, Nevin KP, Ward JE, Woodard TL, Nonnenmann SS, Lovley DR. 2019. Decorating the outer surface of microbially produced protein nanowires with peptides. ACS Synthetic Biology 8:1809-1817.

26. Tan Y, Adhikari RY, Malvankar NS, Ward JE, Woodard TL, Nevin KP, Lovley DR. 2017. Expressing the Geobacter metallireducens PilA in Geobacter sulfurreducens yields pili with exceptional conductivity. mBio 8:e02203-16.

27. Walker DJF, Nevin KP, Nonnenmann SS, Holmes DE, Woodard TL, Ward JE, Rotaru AE, Mcinerney MJ, Lovley DR. 2020. Syntrophus conductive pili demonstrate that common hydrogen-donating syntrophs can have a direct electron transfer option. ISME J 14:837-846.

28. Liu X, Tremblay P-L, Malvankar NS, Nevin KP, Lovley DR, Vargas M. 2014. A Geobacter sulfurreducens strain expressing Pseudomonas aeruginosa type IV pili localizes OmcS on pili but Is deficient in Fe(III) oxide reduction and current production. Appl Environ Microbiol 80:1219-1224.

29. Malvankar NS, Tuominen MT, Lovley DR. 2012. Lack of involvement of c-type cytochromes in long-range electron transport in microbial biofilms and nanowires. Energy Environ Sci 5:8651 - 8659.

30. Mehta T, Coppi MV, Childers SE, Lovley DR. 2005. Outer membrane c-type cytochromes required for $\mathrm{Fe}(\mathrm{III})$ and $\mathrm{Mn}(\mathrm{IV})$ oxide reduction in Geobacter sulfurreducens. Appl Environ Microbiol 71:8634-8641. 
326 31. Walker DJF, Li Y, Meier D, Pinches S, Holmes DE, Smith JA. 2020. Cytochrome OmcS is not essential for long-range electron transport in Geobacter sulfurreducens strain

KN400. bioRixiv:doi: https://doi.org/10.1101/2020.07.22.214791.

32. Liu F, Rotaru A-E, Shrestha PM, Malvankar NS, Nevin KP, Lovley DR. 2015. Magnetite compensates for the lack of a pilin-assoicated c-type cytochrome in extracellular electron exchange. Environ Microbiol 17:648-655.

33. Inoue K, Leang C, Franks AE, Woodard TL, Nevin KP, Lovley DR. 2010. Specific localization of the $c$-type cytochrome OmcZ at the anode surface in current-producing biofilms of Geobacter sulfurreducens. Environ Microbiol Rep 3:211-217.

34. Coppi MV, Leang C, Sandler SJ, Lovley DR. 2001. Development of a genetic system for

35. Zhou Z, López-Domínguez P, Abdullah M, Barber DM, Meng X, Park J, Van Driessche I, Schiffman JD, Crosby AJ, Kittilstved KR, Nonnenmann SS. 2021. Memristive behavior of mixed xxide nanocrystal assemblies. ACS Applied Materials \& Interfaces $13: 21635-21644$. 